\title{
Study of Post Operative Fever in Patients Undergoing Clean Surgeries
}

\author{
${ }^{1}$ Anand R Baid, ${ }^{2}$ Sunil Kumar Shetty, ${ }^{3}$ ThangamVerghese Joshua, ${ }^{4}$ Sunil Kumar \\ Math. \\ ${ }^{1,2,3,4}$ Department of Surgery, Kasturba Medical College, Mangalore (a constituent college of Manipal university)
}

\begin{abstract}
:
Introduction \& Background: Fever is a common complaint in post-operative period in patients undergoing clean surgeries. Fever in postoperative period is the result of infection or SIRS.

This study is aimed at determining the cause of fever in post-operative period in patients with clean surgeries. Duration of study is from March 2012 to July 2013. The common causes of fever such as SIRS, superficial thrombophlebitis, urinary tract infection, respiratory infection, wound infection, medical causes and anaesthesia related causes have been evaluated for and categorised accordingly.

Methods:In this prospective study 623 patients were included. Clinical thermometer was used to chart the temperature. Patients with readings more than 38 degree Celsius at more than two intervals at least 6 hours apart were further evaluated in the form of blood investigations, urine routine and culture, chest $x$-ray, blood sugars, malarial parasite test.

Results: 87 patients had fever $\left(>38^{\circ} \mathrm{C}\right)$ postoperatively. 65 patients had fever in the first 24 hours, 15 patients had fever in 24-48 hours and 7 patients had fever 48 hours postoperatively. In 61 patients (70.12\%) SIRS was detected as a cause of fever, 12 patients (13.8\%) had thrombophlebitis, 7 patients (8.04\%) had URTI, 4 (4.6\%) had wound infection, 1 patient (1.14\%) had UTI and 2 patients (2.3\%) hadmalaria.

Conclusion:Of the 87 patients with postoperative fever, 67\% Patients had aged above 40 years. SIRS is the most common cause of post-operative fever.Superficial thrombophlebitis has been found as a second leading cause for post-operative fever in clean surgeries.Pre-existing co-morbid illness increases the chances of postoperative fever.
\end{abstract}

\section{Introduction}

Fever is rise in the core body temperature above the normal diurnal variations. According to studies of healthy individuals $18-40$ years of age, an a.m. temperature of greater than $98.9^{\circ} \mathrm{F}\left(37.2^{\circ} \mathrm{C}\right)$ or a p.m. temperature of greater than $99.9 \mathrm{~F}\left(37.7^{\circ} \mathrm{C}\right)$ would define a fever. However, there is no exact definition or uniform clinical definition of a significant fever. ${ }^{1}$

Rectal temperature is $0.5^{\circ} \mathrm{C}$ higher than oral temperature and more reliable particularly in mouth breathers or in tachypneic state. Post-operative fever is one of the most common problems seen in surgical wards. It is not limited to any specific type of surgery. ${ }^{2}$ Postoperative fever ranges from $14 \%$ to $91 \%,{ }^{3}$ as per the published data.The variations are because of the definitions and methods of measurement of fever and the patient population of study.

In studies it has been observed that patients undergoing contaminated and dirty surgical procedures were found to have fever postoperatively, with SIRS being the most common cause. ${ }^{3}$

Though several studies in the past had shown various causes of postoperative fever in general surgery, but not specific in scenario like elective and clean general surgeries.

This study aims to identify the causes of postoperative fever in elective and clean general surgeries.

\section{Materials \& Methods}

Prospective study conducted in hospitals affiliated to KMC Mangalore. Study conducted between March 2012 to July2013. During this period a total of 623 patients undergoing elective and clean general surgical procedures were included.

\section{Exclusion criteria:}

Patients undergoing emergency surgical procedures. Patients undergoing cleancontaminated,contaminatedand dirty surgical procedures.

\section{Statistical evaluation}

For statistical calculations, chi square test was used with $\mathrm{p}<0.05$ considered as significant. 


\section{Evaluation:}

Patients who were included in the study underwent general health checkup and investigated in the form ofCompletehemogram, renal function test, liver function test, urine routine test, blood glucose levels, and Chest $\mathrm{X}$-ray, prior to the surgery. All surgeries were elective cases. Intraoperatively patients were observed for type of anaesthesia and any anaesthesia related complications andpostoperatively patients were monitored in the form of vitals examination and wound examination.

Regular progress was recorded during their stay in the hospitalsandpatients having temperature above 38 degree Celsius at more than twointervals at least 6 hours apart were further evaluated in the form of base lineinvestigations for all patients like leucocyte count, c-reactive protein, urine routine examination andmalarial parasite test and specific tests depending on patients symptoms like Chest X-ray, intravenouscannula tip for culture, wound swab for culture and blood glucose level.

Patients having fever in the postoperative period were categorized as per the SIRS criteria of ACCP and Critical care Medicine Guidelines, ${ }^{56}$ into SIRS, Sepsis, Severe sepsis, and Septic shock.

\section{Results}

This is a prospective study of one and a half year duration conducted between March 2012 to July 2013. During this period all patients undergoing elective and clean general surgical procedures were included

In our study of 623 patients, males were 421 (67.6\%) and females were 202 (32.4\%). Mean age of the patients was 47.9 years(range 20 to 70 years)

In the study $66(10.6 \%)$ patients were having pre-existing co-morbid illness.

Fever in post-operative period was observed in 39 patients $(59.1 \%)$.

In patients with post-operative fever, 65(74.7\%) patients had fever within first 24 hrs.

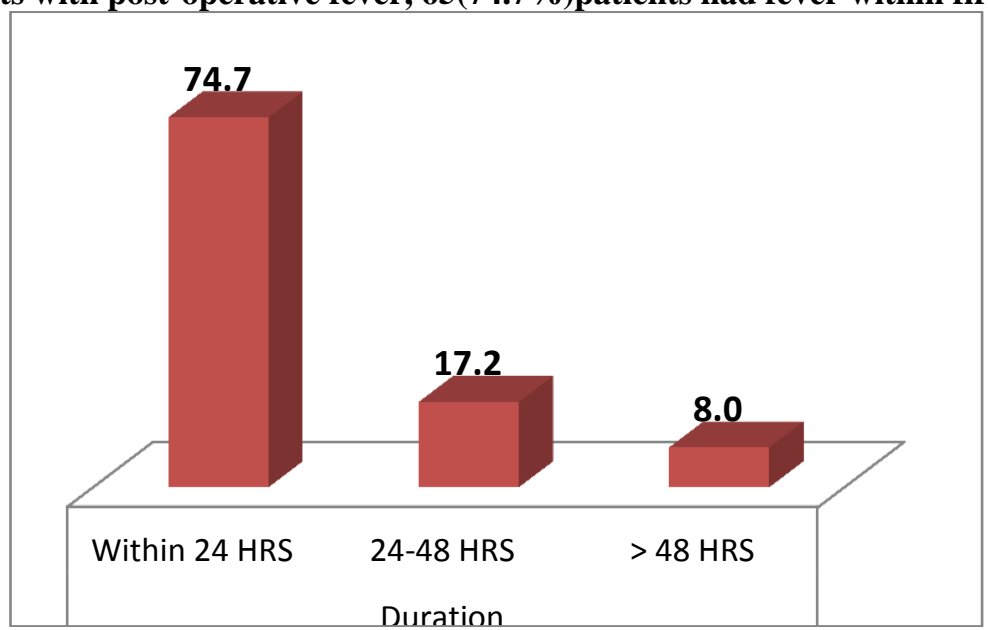

Onset Of Fever Following Surgery

SIRS is the most common cause. $61(70.12 \%)$ patients, with 54(88.5\%)patients had fever in first 24 hours.

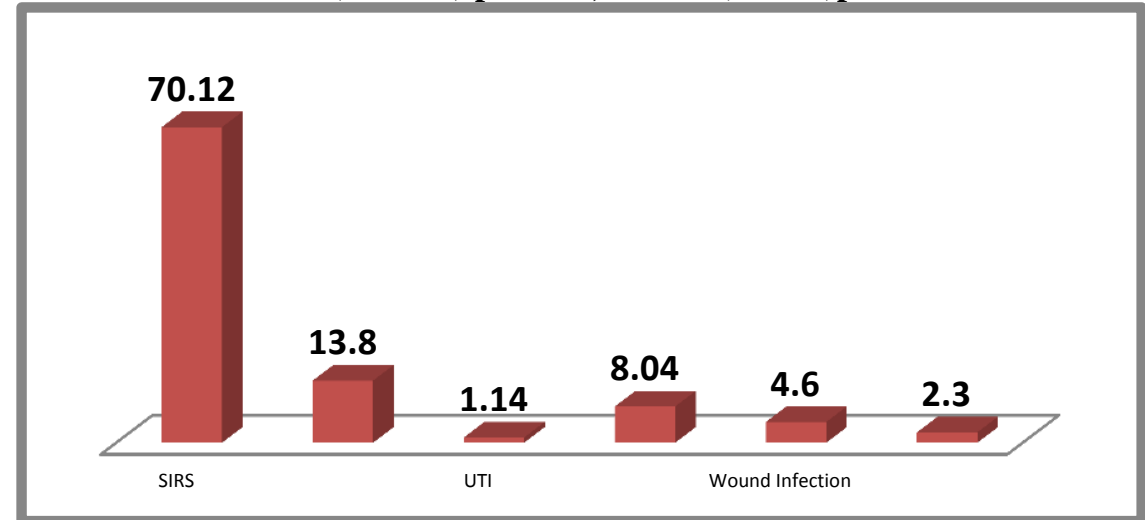

Bar Diagram Showing Various Causes Of Postoperative Fever 
Onset Of Sirs

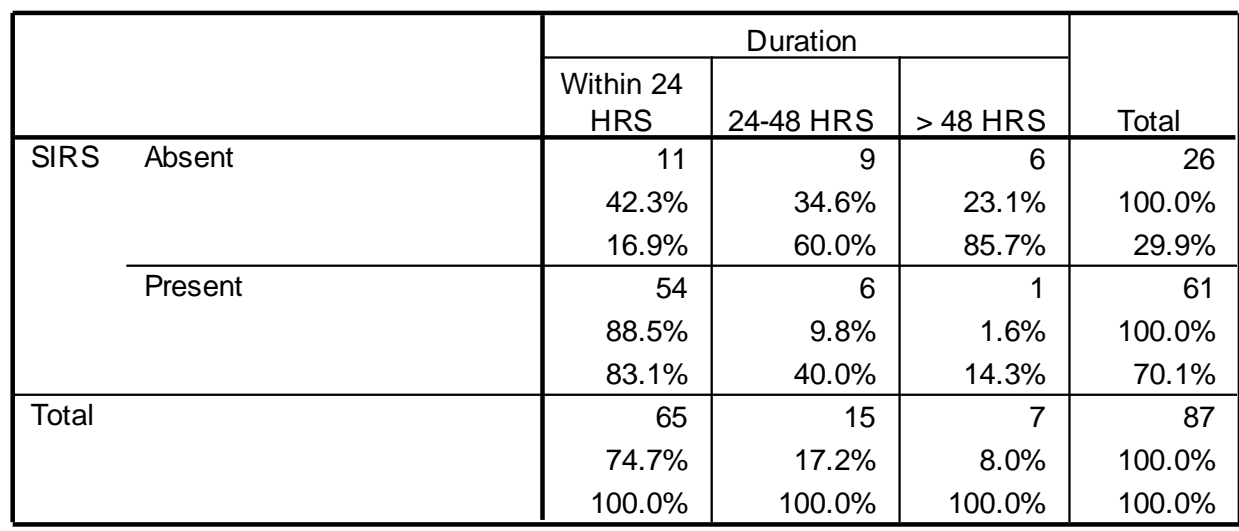

In patients with pre-existing co morbid illness, fever in post-operative period was observed in 39 patients, with onset of fever with in 24 hours was seen in $34(87.2 \%)$ patients.

\begin{tabular}{|ll|r|r|r|r|}
\hline & & \multicolumn{3}{|c|}{ Duration } & \\
\cline { 3 - 6 } & & Within 24 & & \\
& & & \\
& & & & \\
Co MS & $24-48$ HRS & $>48$ HRS & \multicolumn{1}{c|}{ Total } \\
\hline Illness & Absent & 31 & 11 & 6 & 48 \\
& & $64.6 \%$ & $22.9 \%$ & $12.5 \%$ & $100.0 \%$ \\
& & $47.7 \%$ & $73.3 \%$ & $85.7 \%$ & $55.2 \%$ \\
\cline { 2 - 6 } & Present & 34 & 4 & 1 & 39 \\
& & $87.2 \%$ & $10.3 \%$ & $2.6 \%$ & $100.0 \%$ \\
& & $52.3 \%$ & $26.7 \%$ & $14.3 \%$ & $44.8 \%$ \\
\hline Total & 65 & 15 & 7 & 87 \\
& & $74.7 \%$ & $17.2 \%$ & $8.0 \%$ & $100.0 \%$ \\
& & $100.0 \%$ & $100.0 \%$ & $100.0 \%$ & $100.0 \%$ \\
\hline
\end{tabular}

\section{Discussion}

This is a prospective study, conducted during the period between March 2012 to July 2013, in the hospitals affiliated to Kasturba Medical College, Mangalore.

A total number of 623 patients were included.In the entire study group $421(67.6 \%)$ were males and 202 $(32.4 \%)$ were females.

Patients were monitored from the day of admission till the day ofdischarge. During this period patients were evaluated in the form ofgeneral healthcheck upand investigated in the form of , complete hemogram, renal function test, liver function test, urine routine test, blood glucose levels, Chest X-ray prior to the surgery.

The type of anaesthesia was also included in the study to know anaesthesia related complications.

Postoperatively patients were monitored in the form of vitals assessment and wound examination.

Regular progress was recorded, patients having temperature above 38 degree Celsius at more than two intervals at least 6 hours apart were further evaluated inthe form of investigations like leucocyte count, creactive protein, urine routine, malarial parasite test, for all patients and Chest-X-Ray, blood glucose levels, intravenous cannula tip for culture, wound swab for culture, depending on the patients symptoms.

In this study it has been observed that in 61 of 87 (70.12\%) patients having fever postoperatively there was no evidence of infection and 26(29.88\%) patients had evidence of infection.

As per the ACCP and CCM Criteria ${ }^{4}$ it was found that 61(70.12\%) patients had SIRS and 26(29.88\%) patients had Sepsis as a cause of postoperative fever.

In our study no cases of Severe Sepsis and Septic Shock were found.

Studies have shown that early postoperative fever may be caused by a inflammatory response. ${ }^{5}$

In this study it has been observed that superficial thrombophlebitis(13.8\%)appears to be second common cause of fever postoperatively following SIRS. 
Studies have shown that Superficial thrombophlebitis occurs in patients with intravenous cannula, resulting in exit site infections and bacteremia, especially if they were placed emergently under nonsterile conditions. ${ }^{6}$

In this study it has been observed that patients with Pre existing co-morbid Illness $(10.6 \%)$ had increase incidence of postoperative fever $(59.1 \%)$.

Studies have shown that surgical stress may lead to the exacerbation of pre- existing medical condition $^{7}$,such as surgical stress altering the blood glucose levels in diabetes mellitus patients.

In this study it has been observed that wound infection (4.6\%), Medical causes (2.3\%),UTI (1.14\%) as a cause of postoperative fever, are not stastically significant.

Studies have shown that the risk of having a urinary tract infection (UTI) is directly related to the duration of catheterization. ${ }^{6}$ In the study, the type of anaesthesia, and anaesthesia related complications leading to post operative fever is not found.Studies have shown that, the anaesthetic technique used during surgery appears to have minimal effect onpostoperative fever. ${ }^{5}$ URTI leading to fever postoperatively is common after 24 hours of surgery.In the study it has been observed that incidence of post operative fever is more within 24 hours following surgery (74.7\%).Limitations: In our study all patients, who had fever postoperatively were evaluated only with routine baseline investigations and specific investigations were individualized according to patients symptoms.In the study only incidence of various causes of postoperative fever have been studied, further evaluation is required.

\section{Conclusion}

It has been found that fever in the postoperative period is also a common complaint in elective clean surgical procedures.Patients aged above 40 years were found to have high incidence of fever in the postoperative period.SIRS is the most common cause post-operative fever in clean surgeries.Superficial thrombophlebitis has been found as a second leading cause for post-operative fever in clean surgeries.Preexisting co-morbid illness has been shown to increase the chances of postoperative fever.

\section{References}

[1]. Dinarello CA, Gelfand JA. Fever and hyperthermia. In : Harrison's principles of internal medicine. 16th ed. McGrawHill: 2005. p. 104-7.

[2]. Mackowiak PA. Fever, In: Mandell GL, Bennett JE, Polin R, editors. Principles and Practice of infectious diseases. 5th ed. Churchill Livingstone: Philadelphia; 2000. p. 604-21.

[3]. Dellinger EP. Approach to the patient with postoperative fever. In :Gorbach SL, Bartlett JG, Blacklow NR, editors. Infectious diseases. 3rd ed. Lippincott Williams and Wilkins: Philadelphia, PA; 2004. p. 817.

[4]. Sanborn TA, Feldman T: Management strategies for cardiogenic shock. CurrOpinCardiol2004;19:608-612.

[5]. Frank SM, Kluger MJ \& Kunkel SL. Elevated thermostatic setpoint in postoperative patients. Anesthesiology $2000 ; 93: 1426-1431$.

[6]. Weed H, Baddour LM. Postoperative fever. Up to Date; 2011.

[7]. House J, Alexandraki I. Postoperative fever. BMJ Best Practice 2012 